Gut and Liver, Vol. 11, No. 4, July 2017, pp. 481-488

\title{
A Novel Partially Covered Self-Expandable Metallic Stent with Proximal Flare in Patients with Malignant Gastric Outlet Obstruction
}

Naminatsu Takahara ${ }^{1}$, Hiroyuki Isayama ${ }^{1}$, Yousuke Nakai ${ }^{1}$, Shuntaro Yoshida ${ }^{1}$, Tomotaka Saito ${ }^{2}$, Suguru Mizuno ${ }^{3}$, Hiroshi Yagioka ${ }^{4}$, Hirofumi Kogure ${ }^{1}$, Osamu Togawa ${ }^{5}$, Saburo Matsubara ${ }^{1}$, Yukiko Ito $^{6}$, Natsuyo Yamamoto ${ }^{1}$, Minoru Tada ${ }^{1}$, and Kazuhiko Koike ${ }^{1}$

${ }^{1}$ Department of Gastroenterology, Graduate School of Medicine, The University of Tokyo, ${ }^{2}$ Department of Gastroenterology, JR Tokyo General Hospital, ${ }^{3}$ Department of Gastroenterology, Toshiba Hospital, ${ }^{4}$ Department of Gastroenterology, Tokyo Metropolitan Police Hospital, ${ }^{5}$ Department of Gastroenterology, Kanto Central Hospital, and ${ }^{6}$ Department of Gastroenterology, Japanese Red Cross Medical Center, Tokyo, Japan

Background/Aims: Endoscopic placement of self-expandable metal stents (SEMSs) has emerged as a palliative treatment for malignant gastric outlet obstruction (GOO). Although covered SEMSs can prevent tumor ingrowth, frequent migration of covered SEMSs may offset their advantages in preventing tumor ingrowth. Methods: We conducted this multicenter, single-arm, retrospective study at six tertiary referral centers to evaluate the safety and efficacy of a partially covered SEMS with an uncovered large-bore flare at the proximal end as an antimigration system in 41 patients with symptomatic malignant GOO. The primary outcome was clinical success, and the secondary outcomes were technical success, stent dysfunction, adverse events, and survival after stent placement. Results: The technical and clinical success rates were $100 \%$ and $95 \%$, respectively. Stent dysfunctions occurred in 17 patients (41\%), including stent migration in nine $(23 \%)$, tumor ingrowth in one $(2 \%)$, and tumor overgrowth in four (10\%). Two patients (5\%) developed adverse events: one pancreatitis and one perforation. No procedurerelated death was observed. Conclusions: A novel partially covered SEMS with a large-bore flare proximal end was safe and effective for malignant GOO but failed to prevent stent migration. Further research is warranted to develop a covered SEMS with an optimal antimigration system. (Gut Liver 2017;11:481-488)

Key Words: Gastric outlet obstruction; Self expandable metal stent; Endoscopic stent placement

\section{INTRODUCTION}

Malignant gastric outlet obstruction (GO0) is a serious complication which often develops in patients with the advance stage of several types of cancer. Given the poor general condition in patients with malignant G00, endoscopic self-expandable metal stents (SEMS) placement is an attractive alternative to surgical gastrojejunostomy, because endoscopic SEMS placement can provide a rapid relief in obstructive symptoms with lower morbidity and mortality compared to surgical gastrojejunostomy. ${ }^{1-4}$

Although several reports showed the safety and efficacy of covered and uncovered SEMSs for malignant G00, both of them have inherent advantages and disadvantages..$^{5-8}$ Generally, covered SEMS can prevent tumor ingrowth which is the major cause of dysfunction in uncovered SEMS, but is prone to migration. ${ }^{9-11}$ In our previous study, a partially covered, triple-layer SEMS with prolonged uncovered portion at both ends, modified ComVi stent (Taewoong Medical, Gimpo, Korea), showed no tumor ingrowth without increasing the risk of migration. ${ }^{12}$ To further reduce the risk of stent migration, a novel partially covered SEMS with a large-bore flare proximal end, Flared-ComVi stent (Niti-S stent, ComVi type, Flare; Taewoong Medical), was developed. We evaluated the safety and efficacy of this novel SEMS for malignant GOO in this study.

\section{MATERIALS AND METHODS}

This study was a multicenter, single-arm, retrospective study to evaluate the safety and efficacy of the Flared-ComVi stent at

Correspondence to: Hiroyuki Isayama

Department of Gastroenterology, Graduate School of Medicine, The University of Tokyo, 7-3-1 Hongo, Bunkyo-ku, Tokyo 113-8655, Japan

Tel: +81-3-3815-5411 (ext. 33056), Fax: +81-3-3814-0021, E-mail: isayama-tky@umin.ac.jp

Received on May 19, 2016. Revised on August 14, 2016. Accepted on September 27, 2016. Published online February 17, 2017

pISSN 1976-2283 eISSN 2005-1212 https://doi.org/10.5009/gnl16259

() This is an Open Access article distributed under the terms of the Creative Commons Attribution Non-Commercial License (http://creativecommons.org/licenses/by-nc/4.0) which permits unrestricted non-commercial use, distribution, and reproduction in any medium, provided the original work is properly cited. 
Tokyo University Hospital and five affiliated hospitals. Written informed consent was obtained from all patients before the procedure. This study was approved by the local ethical committee of each hospital.

\section{Patients}

Between March 2014 and May 2015, consecutive patients undergoing a Flared-ComVi stent placement for symptomatic malignant G00 were retrospectively studied. Patients with multiple

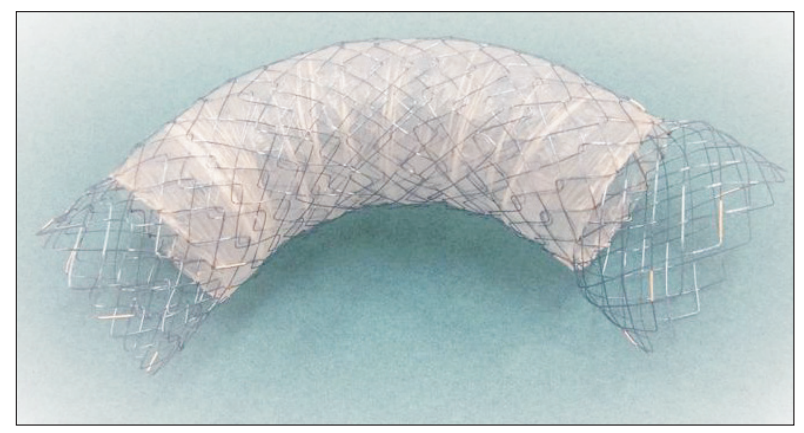

Fig. 1. Flared-ComVi stent. Flared-ComVi stent (Niti-S stent, ComVi type [Flare]; Taewoong Medical) is constructed of a polytetrafluoroethylene (e-PTFE) membrane sandwiched between two uncovered nitinol self-expandable metal stent layers and has a large-bore uncovered proximal flare end ( $25 \mathrm{~mm}$ in diameter and $15 \mathrm{~mm}$ in length). gastrointestinal (GI) tract strictures and patients with a history of previous palliative gastrojejunostomy or a SEMS placement for malignant G00 were excluded.

\section{Flared-ComVi stent}

The flared-ComVi stent (Taewoong Medical) was constructed of a polytetrafluoroethylene (e-PTFE) membrane sandwiched between two uncovered nitinol SEMS layers. The outer uncovered layer was expected to prevent SEMS migration. The flaredComVi stent has low axial force which allows to maintain its bent shape when placed in a tortuous portion of GI tract. ${ }^{13}$ The Flared-ComVi stent expands up to $20 \mathrm{~mm}$ in diameter and has a large-bore uncovered proximal flare end $(25 \mathrm{~mm}$ in diameter and $15 \mathrm{~mm}$ in length) with nonflare uncovered distal end (20 $\mathrm{mm}$ in diameter and $15 \mathrm{~mm}$ in length). The lengths of 80, 100, and $120 \mathrm{~mm}$ are commercially available, with the delivery system of $10 \mathrm{~F}$ (Fig. 1).

\section{Stent placement}

Details of the SEMS placement procedure have been reported previously. ${ }^{6,12}$ Under conscious sedation with intravenous pethidine hydrochloride and diazepam, the procedure was performed using a therapeutic endoscope equipped with 3.7-mm accessory channel (GIF-2T-240 or TJF-260V; Olympus Medical Systems, Tokyo, Japan). The gastroduodenal stricture was passed by us-
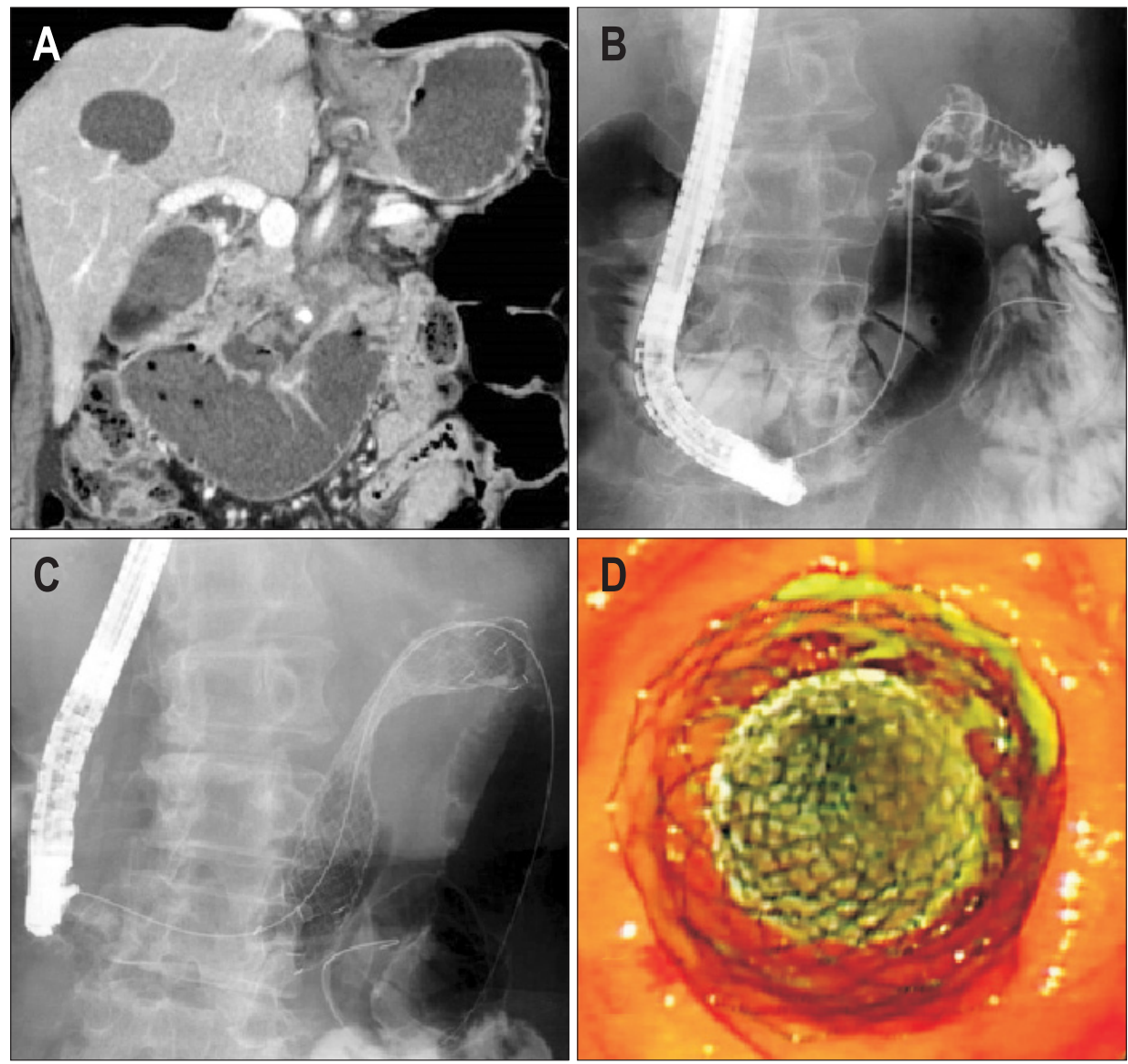

Fig. 2. Stent placement procedure. (A) A computed tomography scan revealed a tight stricture at the third and fourth portions of the duodenum by pancreatic body cancer. (B) A guidewire was passed through the stricture under fluoroscopy. (C) Placement of the Flared-ComVi stent (Taewoong Medical). (D) Endoscopic view of a deployed FlaredComVi stent. 
ing an endoscopic retrograde cholangiopancreatography (ERCP) catheter (ERCP-catheter; MTW Endoskopie Inc., Wesel, Germany) with a 0.035-inch guidewire (Revowave, Piolax Medical Devices, Kanagawa, Japan; Jagwire, Boston Scientific, Natick, MA, USA). After the length of the stricture was assessed by injection of water-soluble radiographic contrast media through a catheter, SEMS length was determined in accordance with the following criteria: (1) the PTFE cover sufficiently covered the stricture to prevent tumor ingrowth and (2) the SEMS was long enough to prevent kinking of the digestive tract at both ends. After the delivery system was advanced through the obstruction over the guidewire via the working channel, the SEMS was carefully deployed under endoscopic and fluoroscopic guidance (Fig. 2). The proximal flare end was placed proximal to the pyloric ring whenever possible. A contrast media was flushed to evaluate patency after the Flared-ComVi stent placement. Oral intake was restarted after no dislocation and sufficient expansion of the SEMS were verified by an abdominal radiography.

\section{Outcomes and definitions}

The data were prospectively collected into our database which was merged at the University of Tokyo Hospital. The primary outcome was clinical success, and the secondary outcomes included technical success, stent dysfunction, adverse events, and survival after stent placement.

Technical success was defined as adequate placement of the SEMS across the stenosis, as confirmed by a combination of endoscopy and fluoroscopy. Clinical success was defined as the relief of obstructive symptoms and/or at least 1 grade of improvement of the gastric outlet obstruction scoring system (G00SS) score within 3 days after SEMS placement. The severity of obstructive symptoms was evaluated with the GOOSS, in which a grade of 0 indicates no oral intake, 1 indicates intake of liquids only, 2 indicates intake of soft solids, and 3 indicates a low residue or a full diet. ${ }^{14}$ Duodenal stenoses were classified according to the location of the obstruction in relation to the major papilla: type I, proximal to and without involvement of the papilla; type II, affecting the second portion of the duodenum and the papilla; type III, distal to and without involvement of the major papilla. ${ }^{15}$

Stent dysfunction was defined as the recurrence of symptoms associated with G00 and an endoscopic examination was performed to evaluate the cause of the SEMS dysfunction when it was feasible for patients. Adverse events were defined and graded according to the lexicon for recommended by the American Society for Gastrointestinal Endoscopy. ${ }^{16}$ Based on timing, adverse events were defined as "early" if it occurred within 14 days and "late" from day 15 onward.

\section{Statistical analysis}

All analyses were performed by using JMP version 11.0 (SAS Institute, Cary, NC, USA). Results are expressed as number (\%) or median (range). Pre- and post-G00SS scores are compared by using the Wilcoxon signed-rank test. Cumulative stent patency and survival after stent placement were analyzed by the Kaplan-Meier method. Stent function was censored when a patient died without stent dysfunction or was alive with a patent stent. The factors which may be associated with stent migration were also evaluated using the Fisher exact test. A p-value $<0.05$ was considered statistically significant.

\section{RESULTS}

\section{Patient characteristics}

Patients' characteristics are shown in Table 1. The etiologies of G00 were pancreatic cancer in 17 (44\%), gastric cancer in 16 (36\%), and other cancers in eight (20\%). Six patients had an anastomotic stricture caused by recurrent tumor. Concurrent biliary obstruction was present in 11 patients (27\%), receiving endoscopic biliary drainage in 10 (trans-papillary biliary drainage in eight; endoscopic ultrasound [EUS]-guided hepaticogastrostomy in two), and percutaneous transhepatic biliary drainage in one. The GOOSS score before SEMS placement was 0 in

Table 1. Patient Characteristics $(n=41)$

\begin{tabular}{lc}
\multicolumn{1}{c}{ Variable } & Value \\
\hline Age, yr & $67(35-89)$ \\
Male sex & $26(63)$ \\
Primary cancer & \\
Pancreatic cancer & $17(44)$ \\
Gastric cancer & $16(36)$ \\
Biliary cancer & $3(8)$ \\
Others & $5(13)$ \\
Karnofsky performance status & \\
100-90 & $6(15)$ \\
$80-70$ & $22(54)$ \\
60-50 & $13(32)$ \\
G00SS score & \\
0 (no oral intake) & $24(59)$ \\
1 (liquids possible) & $11(27)$ \\
2 (soft solids possible) & $6(15)$ \\
Location of obstruction & \\
Type I (proximal to the papilla) & $11(27)$ \\
Type II (involving the papilla) & $19(46)$ \\
Type III (distal to the papilla) & $24(59)$ \\
Anastomosis & $9(22)$ \\
Concurrent biliary obstruction & $10(24)$ \\
Ascites & $6(15)$ \\
Peritoneal dissemination & \\
\hline
\end{tabular}

Data are presented as median (range) or number (\%). GOOSS, gastric outlet obstruction scoring system. 
24 patients (59\%), 1 in 11 (27\%), and 2 in six (15\%).

\section{Technical and clinical success}

As shown in Table 2, technical success was achieved in all 41 patients (100\%), with a median procedure time of 32 minutes (range, 19 to 120 minutes). Stent length was $80 \mathrm{~mm}$ in four patients, $100 \mathrm{~mm}$ in 10 , and $120 \mathrm{~mm}$ in 27 . In nine patients with type II obstruction, ${ }^{15}$ the papilla was masked after SEMS placement. Clinical success was obtained in 39 patients (95\%). Oral intake was restarted at median of 2 days (range, 1 to 9 days) and the G00SS scores were significantly improved after stenting (median of 0 ; range, 0 to 2) versus (median of 3; range, 0 to 3) $(\mathrm{p}<0.01)$. The maximum oral intake after stenting was lowresidual or full diet in 35 patients, soft solids in three, liquids in one, and no oral intake in two. After SEMS placement, 22 (54\%) received systemic chemotherapy including intraperitoneal chemotherapy for peritoneal dissemination. ${ }^{17-19}$

\section{Adverse events}

Two patients (5\%) developed adverse events, including one mild pancreatitis and one perforation. No procedure-related

Table 2. Clinical Outcomes

\begin{tabular}{ll}
\hline \multicolumn{1}{c}{ Variable } & Value \\
\hline Technical success & $41(100)$ \\
Clinical success & $39(95)$ \\
Procedure time, min & $32(19-120)$ \\
Discharge from hospital & $35(85)$ \\
G00SS score after stent placement & \\
$\geq 1$ (liquids possible) & $39(95)$ \\
$\geq 2$ (soft solids possible) & $38(93)$ \\
3 (low-residual or full diet possible) & $35(85)$ \\
Time to restart oral intake, day & $2(1-9)$ \\
Chemotherapy after SEMS placement & $22(54)$ \\
\hline
\end{tabular}

Data are presented as number (\%) or median (range).

G00SS, gastric outlet obstruction scoring system; SEMS, self-expandable metal stent. death was observed. Pancreatitis was observed in a patient who underwent both SEMS placement for malignant GOO and distal biliary obstruction in one session. Because the papilla was not masked by the Flared-ComVi stent, pancreatitis was considered as a consequence of ERCP with biliary stenting. The GI perforation was observed around the fourth portion of duodenum where the distal end of the SEMS was contacted in a patient with pancreatic body cancer receiving chemotherapy at 132 days after SEMS placement. The patient recovered with conservative treatment without any interventions and subsequent GI contrast exam under endoscopy revealed no leakage.

\section{Stent function, reintervention, and survival}

Stent dysfunction and reintervention are summarized in Table 3. During the follow-up period, stent dysfunction occurred in 18 patients (44\%), including stent migration in nine (23\%), tumor overgrowth in four (10\%), and tumor ingrowth in one $(2 \%)$. Among those with stent dysfunction, endoscopic reintervention was successfully performed without any salvage surgical procedure in all 12 patients (29\%): An additional SEMS placement in 11 and balloon dilation in one. The remaining six patients

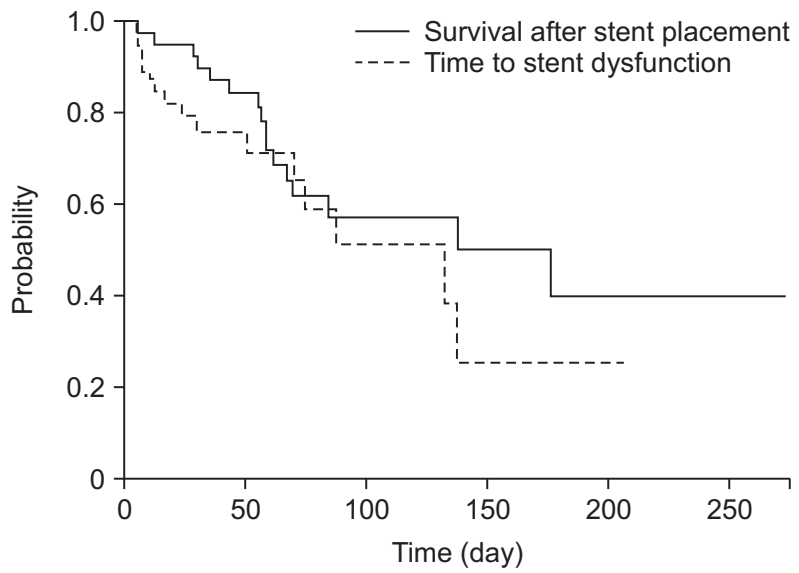

Fig. 3. Cumulative stent patency and survival after stent placement. Kaplan-Meier analysis revealed the median time to stent dysfunction and survival after stent placement of 132 and 176 days, respectively.

Table 3. Stent Dysfunction and Reintervention

\begin{tabular}{lll}
\hline & No. (\%) & \\
\hline Migration & $9(23)$ & 7 Additional SEMS placement (including SEMS removal in 2) \\
& & 2 No additional SEMS placement \\
Tumor overgrowth & $4(10)$ & 3 Additional SEMS placement \\
& $1(2)$ & 1 No intervention \\
Tumor ingrowth & $1(2)$ & 1 No intervention \\
Hyperplasia & $1(2)$ & 1 Bdditional SEMS placement \\
Incomplete stent expansion & $2(5)$ & 2 No intervention \\
Food impaction &
\end{tabular}

SEMS, self-expandable metal stent. 


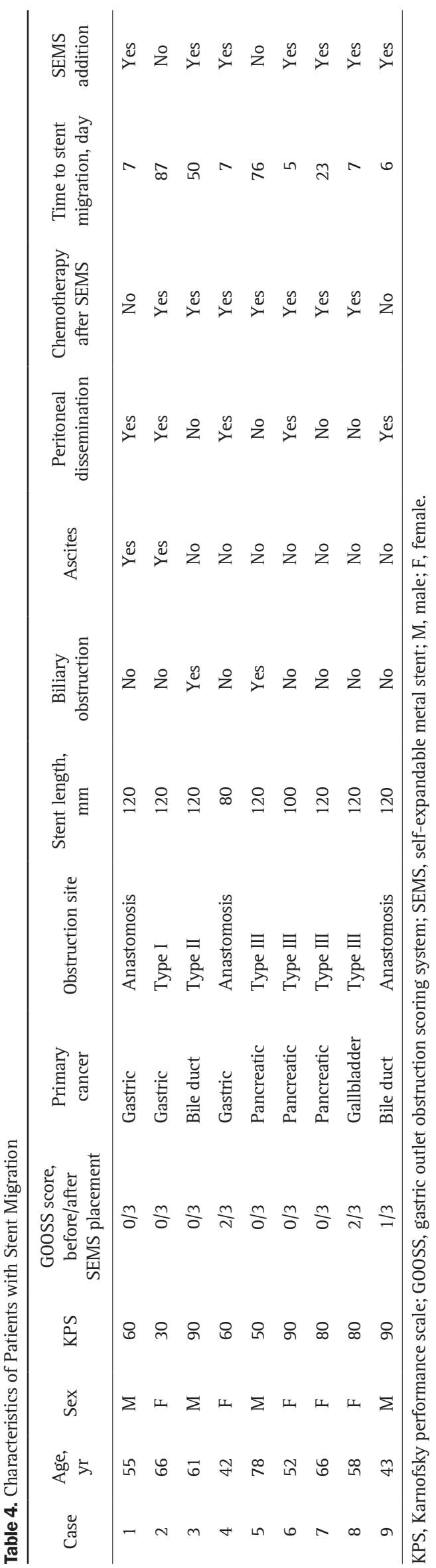

received no endoscopic reintervention because of poor general condition in one, multiple GI strictures in one, and improvement with conservative therapy in four. Cumulative stent patency and survival after stent placement were 132 and 176 days, respectively (Fig. 3).

\section{Migration}

Of note, stent migration occurred in nine patients (23\%), including early migration in five and late migration in four (Table 4). In five patients with early stent migration, all SEMSs were distally migrated within 7 days and additional uncovered SEMSs placement (WallFlex, Boston Scientific; Niti-S, Taewoong Medical) were required (Fig. 4). The remaining four patients with late migration encountered stent migration at 23, 50, 76, and 87 days, respectively. Among these, all SEMSs were spontaneously discharged without stent removal and additional SEMS placement was required only in two patients. Asymptomatic stent migration in the remaining two patients was incidentally diagnosed by computed tomography scan for the evaluation of chemotherapy, and needed no additional SEMS placement.

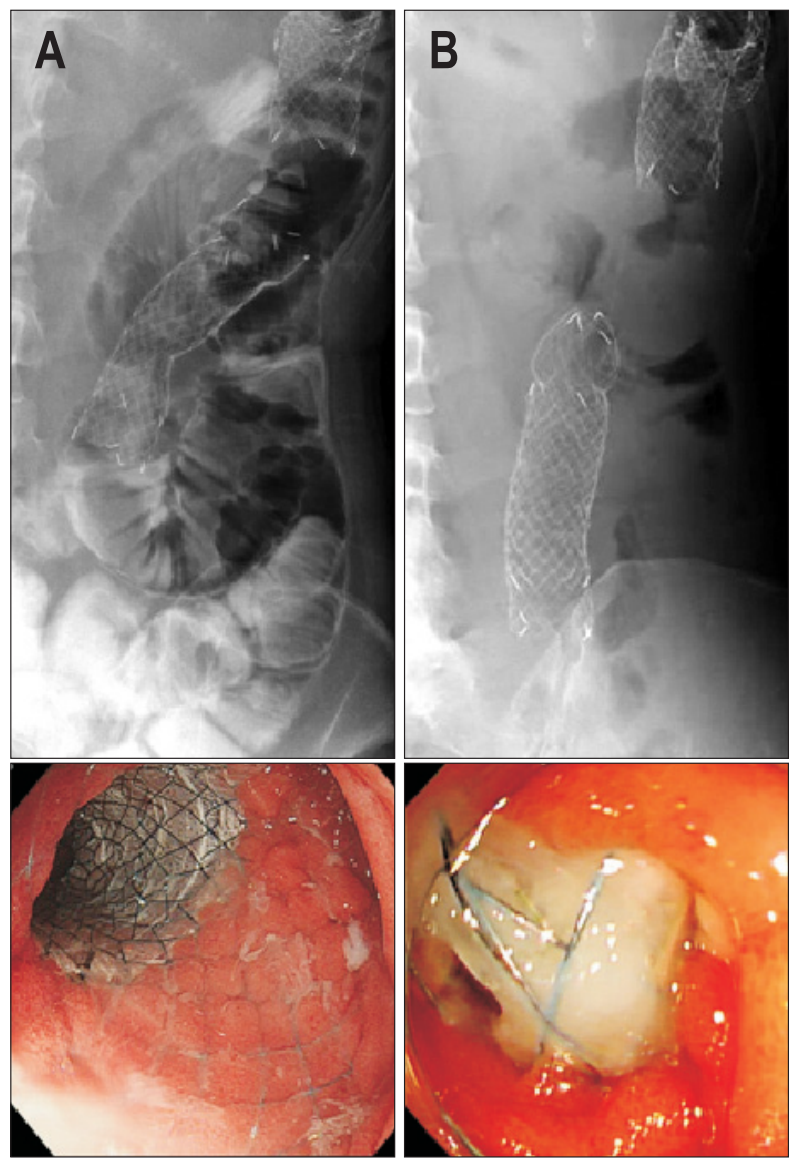

Fig. 4. Case of stent migration. (A) A Flared-ComVi stent (Taewoong Medical) was successfully placed to cover the stricture at the anastomosis site, and the oral flare end appeared to be completely opening. (B) A Flared-ComVi stent was distally migrated with the flare end of collapsed (3 days after stent placement). 
As shown in Table 5, an exploratory analysis showed younger age $<60$ years, ascites, anastomotic obstruction and chemotherapy after SEMS placement tended to be associated with SEMS migration.

\section{DISCUSSION}

This study demonstrated that the newly designed FlaredComVi stent was safe and effective to palliate symptoms in pa-

Table 5. Factors Associated with Stent Migration

\begin{tabular}{|c|c|c|}
\hline Variable & Stent migration & p-value \\
\hline Age, yr & & 0.04 \\
\hline$<60$ & $5 / 9(56)$ & \\
\hline$\geq 60$ & $4 / 32(13)$ & \\
\hline Sex & & 0.29 \\
\hline Female & $5 / 15(33)$ & \\
\hline Male & $4 / 26(15)$ & \\
\hline Karnofsky performance status & & 0.44 \\
\hline$>70$ & $5 / 17(29)$ & \\
\hline$\leq 70$ & $4 / 24(17)$ & \\
\hline G00SS score & & 0.65 \\
\hline 0 & $6 / 24(25)$ & \\
\hline $1-2$ & $3 / 17(18)$ & \\
\hline Primary cancer & & 0.65 \\
\hline Gastroduodenal & $3 / 17(18)$ & \\
\hline Nongastroduodenal cancer & $6 / 24(25)$ & \\
\hline Obstruction site & & 0.19 \\
\hline Anastomosis & $3 / 6(50)$ & \\
\hline Other & $6 / 35(17)$ & \\
\hline Stent length, mm & & 0.49 \\
\hline 120 & $7 / 27(26)$ & \\
\hline $80-100$ & $2 / 14(14)$ & \\
\hline Concurrent biliary obstruction & & 0.72 \\
\hline Yes & $2 / 11(18)$ & \\
\hline No & $7 / 30(23)$ & \\
\hline Ascites & & 0.18 \\
\hline Yes & $2 / 19(11)$ & \\
\hline No & $7 / 22(32)$ & \\
\hline Peritoneal dissemination & & 0.98 \\
\hline Yes & $5 / 23(22)$ & \\
\hline No & $4 / 18(22)$ & \\
\hline Chemotherapy after SEMS placement & & 0.15 \\
\hline Yes & $7 / 21(33)^{*}$ & \\
\hline No & $2 / 20(10)$ & \\
\hline
\end{tabular}

Data are presented as number/total number (\%).

GOOSS, gastric outlet obstruction scoring system; SEMS, self-expandable metal stent.

*Five patients developed stent migration prior to introduction of chemotherapy. tients with malignant G00. However, contrary to our expectations, the large-bore uncovered flare at proximal end could not reduce stent migration.

Recent randomized trials of covered and uncovered SEMSs for malignant GOO demonstrated a significantly lower tumor ingrowth rate in covered SEMSs (1\% to 3\%); however, this advantage was offset by a significantly higher migration rate (13\% to $32 \%$ ), which ultimately resulted in comparable overall stent patency between covered and uncovered SEMSs. ${ }^{9,10,20}$ Therefore, it is desirable to develop a novel SEMS which can overcome stent migration as well as tumor ingrowth. Previously, we reported modified ComVi stent which has long uncovered portion at both ends was associated with decreased risk of stent migration (6.8\%). ${ }^{12}$ Here, Flared-ComVi stent was developed based on modified ComVi stent by adding proximal flare end in order to reduce stent migration further. In this study, however, FlaredComVi stent failed to prevent stent migration (23\%). Although the reasons of a high migration rate in Flared-ComVi stent were unclear, our exploratory analysis showed that age $<60$, ascites, anastomotic obstruction, and chemotherapy after SEMS placement tended to be associated with SEMS migration. A prospective cohort study found a significant association between the use of chemotherapy and stent migration, ${ }^{21}$ but in the current study only two out of nine patients developed stent migration during chemotherapy. Therefore, it is unlikely that reduced tumor burden by chemotherapy was associated with stent migration in our cohort. Rather, the design of the proximal flared end might not be ideal to prevent migration. The radial force of proximal flare end was intentionally designed to be low to prevent perforation, but might be too low to prevent migration and, in addition, the flare can catch food particles and we speculate a large amount food in the flared end might further increase the risk of migration with peristalsis (as shown in Fig. 4). We believe the improvement of antimigration system is important to further prolong stent patency of covered SEMS for G00. One possible option is to develop an ideal flare which has an appropriate size and radial force, preventing migration as well as perforation. The other is to fix stent using clips or suturing devices as demonstrated in esophageal stent. ${ }^{22,23}$

Other than stent migration, this SEMS achieved high technical and clinical success rates. Technical success rate of 100\% and clinical success rate of 95\% were comparable to previous reports: $90 \%$ to $100 \%$ and $80 \%$ to $95 \%$, respectively. ${ }^{5-8,12}$ Patients showed fast relief of symptoms and improved G00SS scores (85\% of patients could have a low residue or a full diet within 5 days). Additionally, we did not encounter severe adverse events in this study. Therefore, Flare-ComVi stent provided safe and effective palliation for G00.

The other disadvantage of covered SEMS is the inherent risk of pancreatitis and cholangitis when placed across the ampulla. In this study, no pancreatitis or cholangitis were observed among nine patients whose ampulla was masked by the stent. 
A previous study also indicated that covered SEMS is unlikely to obstruct bile flow, even if placed across the ampulla. ${ }^{24}$ However, one possible drawback to this is that endoscopic access to biliary system through ampulla becomes nearly impossible in patients developing malignant biliary obstruction after a covered SEMS placement for G00. Because we previously reported duodenal tumor invasion was a significant risk factor for early biliary SEMS dysfunction, ${ }^{25}$ EUS-guided transmural approach is considered to be a preferable option in patients who required biliary drainage as well as GI stenting. ${ }^{26,27}$

This study has some limitations including its retrospective design with a small number of patients. In addition, the study lacked a control group for comparison. However, our previous report of Modified-ComVi stent can be a historical control because all the difference with Flared-ComVi stent is its proximal flare end, and we found the flared end failed to prevent stent migration.

In conclusion, a Flared-ComVi stent was safe and effective for malignant G00, but the flared end failed to prevent stent migration, contrary to our expectations. This finding should be taken into account before this stent is introduced into the clinical practice, and we believe further improvement of antimigration system is warranted.

\section{CONFLICTS OF INTEREST}

No potential conflict of interest relevant to this article was reported.

\section{REFERENCES}

1. Jeurnink SM, Steyerberg EW, van Hooft JE, et al. Surgical gastrojejunostomy or endoscopic stent placement for the palliation of malignant gastric outlet obstruction (SUSTENT study): a multicenter randomized trial. Gastrointest Endosc 2010;71:490-499.

2. Chandrasegaram MD, Eslick GD, Mansfield CO, et al. Endoscopic stenting versus operative gastrojejunostomy for malignant gastric outlet obstruction. Surg Endosc 2012;26:323-329.

3. Khashab M, Alawad AS, Shin EJ, et al. Enteral stenting versus gastrojejunostomy for palliation of malignant gastric outlet obstruction. Surg Endosc 2013;27:2068-2075.

4. Zheng B, Wang X, Ma B, Tian J, Jiang L, Yang K. Endoscopic stenting versus gastrojejunostomy for palliation of malignant gastric outlet obstruction. Dig Endosc 2012;24:71-78.

5. Maetani I, Isayama H, Mizumoto Y. Palliation in patients with malignant gastric outlet obstruction with a newly designed enteral stent: a multicenter study. Gastrointest Endosc 2007;66:355-360.

6. Sasaki T, Isayama H, Maetani I, et al. Japanese multicenter estimation of WallFlex duodenal stent for unresectable malignant gastric outlet obstruction. Dig Endosc 2013;25:1-6.

7. van den Berg MW, Haijtink S, Fockens P, et al. First data on the Evolution duodenal stent for palliation of malignant gastric outlet obstruction (DUOLUTION study): a prospective multicenter study. Endoscopy 2013;45:174-181.

8. van Hooft JE, van Montfoort ML, Jeurnink SM, et al. Safety and efficacy of a new non-foreshortening nitinol stent in malignant gastric outlet obstruction (DUONITI study): a prospective, multicenter study. Endoscopy 2011;43:671-675.

9. Kim CG, Choi IJ, Lee JY, et al. Covered versus uncovered selfexpandable metallic stents for palliation of malignant pyloric obstruction in gastric cancer patients: a randomized, prospective study. Gastrointest Endosc 2010;72:25-32.

10. Lee KM, Choi SJ, Shin SJ, et al. Palliative treatment of malignant gastroduodenal obstruction with metallic stent: prospective comparison of covered and uncovered stents. Scand J Gastroenterol 2009;44:846-852.

11. Maetani I, Mizumoto Y, Shigoka H, et al. Placement of a triplelayered covered versus uncovered metallic stent for palliation of malignant gastric outlet obstruction: a multicenter randomized trial. Dig Endosc 2014;26:192-199.

12. Isayama $\mathrm{H}$, Sasaki T, Nakai $\mathrm{Y}$, et al. Management of malignant gastric outlet obstruction with a modified triple-layer covered metal stent. Gastrointest Endosc 2012;75:757-763.

13. Isayama H, Nakai $Y$, Toyokawa $Y$, et al. Measurement of radial and axial forces of biliary self-expandable metallic stents. Gastrointest Endosc 2009;70:37-44.

14. Piesman M, Kozarek RA, Brandabur JJ, et al. Improved oral intake after palliative duodenal stenting for malignant obstruction: a prospective multicenter clinical trial. Am J Gastroenterol 2009;104:2404-2411.

15. Mutignani M, Tringali A, Shah SG, et al. Combined endoscopic stent insertion in malignant biliary and duodenal obstruction. Endoscopy 2007;39:440-447.

16. Cotton PB, Eisen GM, Aabakken L, et al. A lexicon for endoscopic adverse events: report of an ASGE workshop. Gastrointest Endosc 2010;71:446-454.

17. Nakai $\mathrm{Y}$, Isayama $\mathrm{H}$, Saito $\mathrm{K}$, et al. A phase I trial of gemcitabine, S-1 and LV combination (GSL) therapy in advanced pancreatic cancer. Cancer Chemother Pharmacol 2014;74:911-915.

18. Takahara N, Isayama H, Nakai Y, et al. Intravenous and intraperitoneal paclitaxel with S-1 for refractory pancreatic cancer with malignant ascites: an interim analysis. J Gastrointest Cancer 2014;45:307-311.

19. Ishigami H, Kitayama J, Kaisaki S, et al. Phase II study of weekly intravenous and intraperitoneal paclitaxel combined with S-1 for advanced gastric cancer with peritoneal metastasis. Ann Oncol 2010;21:67-70.

20. Lim SG, Kim JH, Lee KM, et al. Conformable covered versus uncovered self-expandable metallic stents for palliation of malignant gastroduodenal obstruction: a randomized prospective study. Dig Liver Dis 2014;46:603-608.

21. Kim JH, Song HY, Shin JH, et al. Metallic stent placement in the palliative treatment of malignant gastroduodenal obstructions: prospective evaluation of results and factors influencing outcome 
in 213 patients. Gastrointest Endosc 2007;66:256-264.

22. Yang J, Siddiqui AA, Kowalski TE, et al. Esophageal stent fixation with endoscopic suturing device improves clinical outcomes and reduces complications in patients with locally advanced esophageal cancer prior to neoadjuvant therapy: a large multicenter experience. Surg Endosc. Epub 2016 Aug 5. https://doi.org/10.1007/ s00464-016-5131-3.

23. Diana M, Swanström LL, Halvax P, et al. Esophageal covered stent fixation using an endoscopic over-the-scope clip: mechanical proof of the concept and first clinical experience. Surg Endosc 2015;29:3367-3372.

24. Kim SY, Song HY, Kim JH, et al. Bridging across the ampulla of
Vater with covered self-expanding metallic stents: is it contraindicated when treating malignant gastroduodenal obstruction? J Vasc Interv Radiol 2008;19:1607-1613.

25. Hamada T, Isayama H, Nakai $Y$, et al. Duodenal invasion is a risk factor for the early dysfunction of biliary metal stents in unresectable pancreatic cancer. Gastrointest Endosc 2011;74:548-555.

26. Hamada T, Isayama $H$, Nakai $Y$, et al. Transmural biliary drainage can be an alternative to transpapillary drainage in patients with an indwelling duodenal stent. Dig Dis Sci 2014;59:1931-1938.

27. Park DH. Endoscopic ultrasonography-guided hepaticogastrostomy. Gastrointest Endosc Clin N Am 2012;22:271-280. 